A new non-colloidal suspension model

\title{
A new rate-independent tensorial model for suspensions of non-colloidal rigid particles in Newtonian fluids
}

\author{
O. Ozenda, ${ }^{1,2}$ P. Saramito, ${ }^{1, a)}$ and G. Chambon ${ }^{2}$ \\ 1) Lab. J. Kuntzmann - CNRS and Grenoble university, \\ CS 40700 - 38058 Grenoble cedex 9 - France \\ ${ }^{2)}$ Grenoble Alpes University - IRSTEA - UR ETGR, \\ 2 rue de la Papeterie - St-Martin-d'Hères 38402 - France
}

(Dated: 24 April 2018)

\section{Synopsis}

We propose a new, minimal tensorial model attempting to clearly represent the role of microstructure on the viscosity of non-colloidal suspensions of rigid particles. Qualitatively, this model proves capable of reproducing several of the main rheological trends exhibited by concentrated suspensions: anisotropic and fore-aft asymmetric microstructure in simple shear and transient relaxation of the microstructure towards its stationary state. The model includes only few constitutive parameters, with clear physical meaning, that can be identified from comparisons with experimental data. Hence, quantitative predictions of the complex transient evolution of apparent viscosity observed after shear reversals are reproduced for a large range of volume fractions. Comparisons with microstructural data shows that not only the depletion angle, but the pair distribution function, are well predicted. To our knowledge, it is the first time that a microstructure-based rheological model is successfully compared to such a wide experimental dataset.

\section{INTRODUCTION}

Despite the apparent simplicity of the system, concentrated suspensions of non-colloidal, rigid spheres in a Newtonian fluid display a rich and complex rheological behavior [1-3]. In the inertialess limit (zero Reynolds number), particle dynamics is essentially governed by hydrodynamic interactions since lubrication forces prevent, in principle, direct contacts. Linearity and reversibility of Stokes equation then lead to expect that the macroscopic behavior of the suspension should remain Newtonian. Thus, numerous investigations documented the increase of the effective steady-state viscosity of suspensions with particle volume fraction $\phi[3-5]$. However, a wealth of experimental evidence also showed the existence of non-Newtonian rheological effects as soon as $\phi$ exceeds 0.2 , typically. One of the most prominent examples is the the existence of transient viscosity drops upon reversal of the shearing direction [6-8]. There is nowadays a general agreement to relate these non-Newtonian characteristics to flow-induced changes in the microstructure of the suspension $[3,9,10]$. The pair distribution function $g(\boldsymbol{x})$, i.e. the likelihood of finding pairs of particles at a separation vector $\boldsymbol{x}$, has been shown to become anisotropic and lose fore-aft symmetry under shear, with development of preferential concentration and depletion orientations that depend on the volume fraction $\phi$ [11]. This asymmetry of the microstructure is the hallmark of a loss of reversibility of the system that, again, contradicts expectations based on Stokes equation. Although the precise mechanisms remain to be elucidated, it is generally interpreted as resulting from chaotic dynamics induced by the nonlinearity of the multi-body hydrodynamic interactions [12], and/or from even weak perturbations of the hydrodynamic interactions by non-hydrodynamic near-contact forces $[13,14]$. Note that the asymmetric microstructure, and the associated normal stresses, are also at the origin of the cross-stream particle migration process observed in these suspensions when the shear rate is heterogeneous $[15,16]$.

\footnotetext{
a) Electronic mail: Pierre.Saramito@imag.fr
} 
Since the pioneering work of Einstein [17], most rheological models for suspensions assume an additive decomposition of the total Cauchy stress tensor $\boldsymbol{\sigma}$ into fluid and particle contributions $[2,17,18]$. This decomposition naturally arises from mixture theories in which macroscopic quantities are obtained from averages over both phases [19-21]. While the fluid contribution is simply given by a Newtonian model (with the viscosity of the interstitial fluid), closure relations are needed to express the particle stresses. Schematically, two groups of models are found in the literature. The first group encompasses purely macroscopic approaches that do not contain explicit reference to the suspension microstructure, apart from the volume fraction $\phi$. The most popular representative of this class is the suspension balance model (SBM), introduced in 1994 by Nott and Brady [22] (see also [15, $23]$ ), in which particle stresses are expressed as the sum of a shear and a normal term that are both linear in shear rate, with corresponding shear and normal viscosities given by empirical functions of $\phi$. By construction, SBM well reproduces experimental rheological measurements obtained in stationary shear. It also leads to realistic predictions concerning particle migration when the particle normal stresses are used as the driver of the migration flux, even if this approach has been questioned [21, 24]. However, as a counterpart for its simplicity, this model is devoid of any time or strain scale, and therefore unable to account for transients observed during shear reversal experiments. In addition, earlier versions were not invariant by changes of reference frame, although an ad hoc frame-invariant extension has been proposed [16].

In the second group of models, particle stress is made explicitly dependent on the microstructure through the consideration of a local conformation tensor that is inspired from the orientation distribution tensor defined for dilute fiber suspensions (see e.g. [25, 26]). The conformation tensor, denoted $\boldsymbol{b}_{\boldsymbol{e}}$ in this paper, is a second-order symmetric positive definite tensor describing microstructure anisotropy. Hand [27] formulated a general representation theorem for the total Cauchy stress tensor $\boldsymbol{\sigma}$ in term of the conformation tensor $\boldsymbol{b}_{e}$ and the deformation rate tensor $\dot{\gamma}$. This general representation should be closed by a constitutive equation for the evolution of the conformation tensor $\boldsymbol{b}_{e}$. An important constraint is that the characteristic time associated to the evolution of $\boldsymbol{b}_{e}$ must scale inversely with the deformation rate $|\dot{\gamma}|$ in order to ensure strain-scaling and rate-independence of the transients, as observed experimentally (see e.g. [8]) and imposed by dimensional analysis [9, 28]. Note that the rate-independence constraint leads to constitutive equations that are formally similar to hypo-elastic models (see e.g. [29]). For concentrated suspensions of spherical particles, Phan-Thien [30] proposed a differential constitutive equation for the conformation tensor, that led to prediction qualitatively in agreement with time-dependent experimental observations in shear reversal $[6,7,31]$. The structural unit used to define the conformation tensor was taken as the unit vector $\boldsymbol{n}$ joining two neighboring particles, thereby encoding a direct connection with the pair distribution function $g(\boldsymbol{x})$. Later, Phan-Thien et al. [32, 33] went further with a micro-macro model inspired from statistical mechanics for the constitutive equation of the conformation tensor, but no quantitative comparisons were obtained. In 2006, Goddard [28] revisited this approach, and proposed a model involving twelve material parameters and two tensors for describing the anisotropy. By a systematic fitting procedure of the parameters, he obtained numerical results in quantitative agreement with shear reversal experiments [7, 31]. Also in 2006, Stickel et al. [34] (see also [35, 36]) defined the conformation tensor on the base of particle mean free path, and simplified the expression of the stress to be linear in the deformation rate and the conformation tensor. Their model nevertheless also involves 13 free parameters. These authors obtained numerical results in qualitative agreement with a shear reversal experiment $[7,31]$, but failed to obtain quantitative comparisons. In contrast with SBM model, all these tensorial models are, by construction, frame-invariant and potentially applicable to arbitrary flow geometries and conditions. As for polymer models, normal stress differences naturally arise from the use of some objective derivative of the conformation tensor $\boldsymbol{b}_{e}$ (see [37]). The time-dependent relaxation of this tensor, representing microstructure evolution, leads to transient responses when the loading is varied. Nevertheless, these microstructure-based models are still rather complex, and the identification of parameters is generally not obvious. 
This paper is a contribution to an ongoing effort for the development of more tractable microstructure-based rheological models. With the least number of adjustable parameters, the proposed model is relatively simple, yet capable of accounting both for the macroscopic non-Newtonian rheological features of non-colloidal suspensions and for the rateindependent evolution of the microstructure. In particular, this model is able to describe the experimentally observed anisotropic effects expressed by the pair-distribution function. It also qualitatively and quantitatively agrees, for a wide range of volume fraction, with experimental data for time-dependent shear reversals.

The outline of the paper is as follows. The second section concerns model statement. The third section turns to predictions in stationary shear and validation against experimental data for microstructure anisotropy and depletion angle. Section 4 deals with time-dependent flows, specifically shear reversals, and present comparisons with experiments for the apparent viscosity. Finally, the last section develops a discussion and a conclusion.

\section{MATHEMATICAL MODEL}

\section{A. Rheological model}

As illustrated in Fig. 1, a key feature of the microstructure of sheared suspensions is the existence of preferential directions along which the average inter-distance between particles varies: particles are closer along the compression axis, and farther apart along the depletion axis. In inertialess systems, these preferential directions depend on the concentration $\phi$, but not on the deformation rate $|\dot{\gamma}|$ (see [11]). The rheological model developed in this work is purely macroscopic, and hence no attempt is made at deriving a microscopic evolution equation for the microstructure. However, to clarify the physical meaning of the conformation tensor $\boldsymbol{b}_{e}$ used in the sequel, and to provide a direct link with the microstructure, the following definition is proposed:

$$
\boldsymbol{b}_{e}=d_{0}^{2}\langle\boldsymbol{\ell} \otimes \boldsymbol{\ell}\rangle^{-1},
$$

where $\boldsymbol{\ell}$ is the branch vector joining the centers of two neighboring particles, and $d_{0}$ is the average distance between neighboring particle centers in an isotropic configuration at rest. In what follows, the isotropic configuration at rest will be referred to as the reference configuration. In concentrated suspensions, $d_{0}$ is close to $2 a$, where $a$ denotes particle radius, since particles are in near-contact. The choice of an inverse relation between $\boldsymbol{b}_{e}$ and $\langle\boldsymbol{\ell} \otimes \boldsymbol{\ell}\rangle$ in (1) is motivated by the wish to have a conformation tensor whose largest principal direction is aligned with the depletion axis of the microstructure (Fig. 1). The use of $\boldsymbol{\ell}$ as the main microstructural unit is notably different from the approach followed in most earlier studies, which define a fabric tensor $\langle\boldsymbol{n} \otimes \boldsymbol{n}\rangle$ based on the unit vector joining neighboring particles, $\boldsymbol{n}=\boldsymbol{\ell} /|\boldsymbol{\ell}|[3,28,32,33,38]$. In particular, while the trace of the tensor $\langle\boldsymbol{n} \otimes \boldsymbol{n}\rangle$ is by construction equal to one, the $\langle\boldsymbol{\ell} \otimes \boldsymbol{\ell}\rangle$ tensor and the present $\boldsymbol{b}_{e}$ conformation tensor do not have such a constraint, and thus present additional degrees of freedom.

To couple the conformation tensor $\boldsymbol{b}_{e}$ with a rheological model, we introduce a deformation $\gamma_{e}$ defined as:

$$
\gamma_{e}=\boldsymbol{b}_{e}-\boldsymbol{I}
$$

where $\boldsymbol{I}$ is the identity tensor. For an isotropic microstructure at rest, i.e. the reference configuration, we have $\boldsymbol{b}_{e}=\boldsymbol{I}$, and thus $\gamma_{e}=0$. Hence, $\gamma_{e}$ can be interpreted as the average deformation of the local cages formed by neighboring particles, with respect to the reference configuration. Note that a similar concept of cages formed by nearest neighbors was already introduced by [34]. We then assume that the total deformation of the suspension $\gamma$ can be decomposed into the sum of the cage deformation $\gamma_{e}$ and of a viscous deformation $\gamma_{v}$, which represents the global rearrangements of neighboring particles through the flow:

$$
\gamma=\gamma_{e}+\gamma_{v}
$$



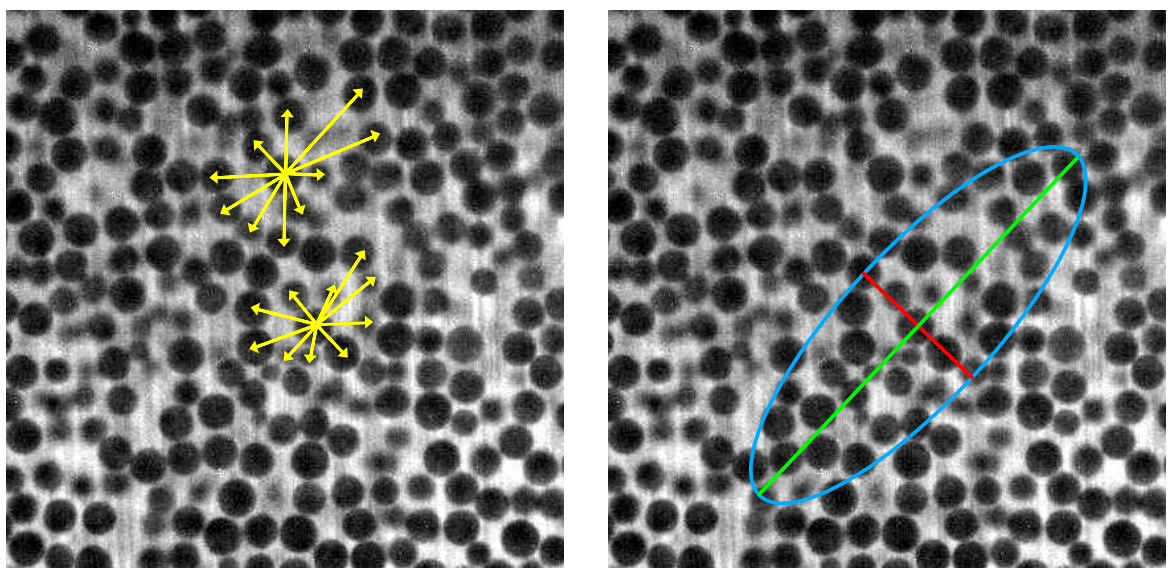

FIG. 1. (left) Schematic representation of the branch vectors $\ell$ joining neighboring particles in a sheared suspension. (right) Representation of the local conformation tensor $\boldsymbol{b}_{e}$ by an ellipse. The compression axis is in red and the depletion axis is in green. Background photography taken from [39], Fig. $4.7(\phi=0.55)$.

The next step is to write a constitutive equation for the variable $\gamma_{e}$. To that aim, we define a local stress, denoted as $\tau_{e}$, and assume the following relation between $\boldsymbol{\tau}_{e}$ and $\boldsymbol{\gamma}_{e}$ :

$$
\tau_{e}=\eta_{g}|\dot{\gamma}| \gamma_{e}
$$

where the tensorial norm $|\boldsymbol{\xi}|$ is defined as $|\boldsymbol{\xi}|=[(1 / 2) \boldsymbol{\xi}: \boldsymbol{\xi}]^{1 / 2}$ for any second-order tensor $\boldsymbol{\xi}$. Observe that this expression is linear with respect to $\gamma_{e}$ and involves a factor $\eta_{g}|\dot{\gamma}|$ that ensures rate-independence of the constitutive relation. Here, $\eta_{g}$ is a constant coefficient with the dimension of a viscosity. This local stress $\tau_{e}$ is also assumed to be linearly related to the rate of viscous deformation $\dot{\gamma}_{v}$ through:

$$
\tau_{e}=\eta_{e} \dot{\gamma}_{v}
$$

where $\eta_{e} \geqslant 0$ is an associated viscosity. Finally, differentiating (2), replacing $\dot{\gamma}_{v}$ from (4) and using (3), the following linear differential equation is obtained for $\gamma_{e}$ :

$$
\dot{\gamma}_{e}+\frac{\eta_{g}}{\eta_{e}}|\dot{\gamma}| \gamma_{e}=\dot{\gamma}
$$

The previous constitutive equation is completed by the following expression for the total Cauchy stress tensor of the suspension:

$$
\begin{aligned}
\boldsymbol{\sigma} & =-p_{f} \boldsymbol{I}+\eta \dot{\boldsymbol{\gamma}}+\boldsymbol{\tau} \\
\text { where } \quad & \boldsymbol{\tau}=\eta_{g}|\dot{\gamma}| \boldsymbol{\gamma}_{e}+\eta_{d}\left(\boldsymbol{\gamma}_{e} \otimes \boldsymbol{\gamma}_{e}\right): \dot{\gamma}
\end{aligned}
$$

The first term involves $p_{f}$, the pressure in the fluid phase. The second term represents the base viscosity of the suspension, expressed here by $\eta \geqslant 0$. Finally, the third term represents the microstructure stress $\tau$. This microstructure stress itself expresses as the sum of the local stress $\tau_{e}$ and of a quadratic term with respect to $\gamma_{e}$. Such quadratic terms commonly derive from the closure of a fourth-order structure tensor in statistical micro-macro models for spherical or fiber suspensions (see e.g. $[25,26]$ ). It involves an additional parameter $\eta_{d}$ with the dimension of a viscosity. Note that this last term writes equivalently $\eta_{d}\left(\dot{\gamma}: \gamma_{e}\right) \gamma_{e}$ and, thus, the two tensors $\boldsymbol{\tau}$ and $\gamma_{e}$ are co-linear and share the same eigensystem. The influence of this additional quadratic contribution will be analyzed in the following.

In the present model, the total stress is thus split into the stress that would be observed for an isotropic microstructure (the base viscosity), and the stress induced by the anisotropic 
arrangement of the microstructure (represented by $\boldsymbol{\tau}$ ). This stress decomposition could appear similar to the decomposition $\boldsymbol{\sigma}=\boldsymbol{\sigma}_{f}+\boldsymbol{\sigma}_{p}$ between fluid $\boldsymbol{\sigma}_{f}$ and particle $\boldsymbol{\sigma}_{p}$ stresses, used in classical mixture theories [20] and in most suspensions models, such as SBM [10, 22]. It shall be emphasized however that, in our approach, the base viscosity $\eta$ also contains a contribution from the particle phase. More precisely, for the present model, we have $\boldsymbol{\sigma}_{f}=-p_{f} \boldsymbol{I}+\eta_{0} \dot{\gamma}$ and $\boldsymbol{\sigma}_{p}=\left(\eta-\eta_{0}\right) \dot{\gamma}+\boldsymbol{\tau}$, where $\eta_{0}$ is the viscosity of the suspending fluid. Roughly, the base viscosity $\eta$ can be seen as accounting for long-range hydrodynamic interactions between particles, while the microstructure stress $\boldsymbol{\tau}$ accounts for short-range, hydrodynamic and contact, interactions. As a consequence, we expect all the parameters of the model, including $\eta, \eta_{e}, \eta_{g}$ and $\eta_{d}$ to depend on volume fraction $\phi$. Finally, also recall that $\operatorname{tr}\left(\gamma_{e}\right)$, and thus $\operatorname{tr}(\boldsymbol{\tau})$, are not necessarily zero, such that the microstructure stress $\boldsymbol{\tau}$ may also contribute to the total pressure $p=-(1 / 3) \operatorname{tr}(\boldsymbol{\sigma})$ of the suspension. Indeed, $p=p_{f}+p_{p}$ with $p_{p}=-(1 / 3) \operatorname{tr}\left(\boldsymbol{\sigma}_{p}\right)=-(1 / 3) \operatorname{tr}(\boldsymbol{\tau})$, as the mixture is assumed to be isochoric with $\operatorname{tr}(\dot{\gamma})=0$.

The time derivative $\dot{\gamma}_{e}$ is given by the upper-convected tensor derivative (see e.g. [37], chap 4), denoted hereafter $\mathscr{D} \gamma_{e} / \mathscr{D} t$ :

$$
\frac{\mathscr{D} \boldsymbol{\gamma}_{e}}{\mathscr{D} t}=\frac{\partial \boldsymbol{\gamma}_{e}}{\partial t}+(\boldsymbol{u} . \nabla) \boldsymbol{\gamma}_{e}-\nabla \boldsymbol{u} \boldsymbol{\gamma}_{e}-\boldsymbol{\gamma}_{e} \nabla \boldsymbol{u}^{T}
$$

The deformation rate $\dot{\gamma}$ is identified to two times the symmetric part of the velocity gradient tensor $D(\boldsymbol{u})=\left(\nabla \boldsymbol{u}+\nabla \boldsymbol{u}^{T}\right) / 2$, where $\boldsymbol{u}$ is the velocity field of the mixture. The constitutive equations thus become:

$$
\begin{aligned}
& \frac{\mathscr{D} \boldsymbol{\gamma}_{e}}{\mathscr{D} t}+\alpha|2 D(\boldsymbol{u})| \boldsymbol{\gamma}_{e}=2 D(\boldsymbol{u}) \\
& \boldsymbol{\sigma}=-p_{f} \boldsymbol{I}+2 \eta D(\boldsymbol{u})+\eta_{e}\left\{\alpha|2 D(\boldsymbol{u})|+\beta\left(D(\boldsymbol{u}): \boldsymbol{\gamma}_{e}\right)\right\} \boldsymbol{\gamma}_{e}
\end{aligned}
$$

where $\alpha=\eta_{g} / \eta_{e}$ and $\beta=2 \eta_{d} / \eta_{e}$ are dimensionless parameters. These constitutive equations can be seen as a rate-independent variant of a viscoelastic Oldroyd [40] model with an additional quadratic term for the total stress. Rate-independence is guaranteed by the the use of an "effective elastic modulus" $\eta_{g}|\dot{\gamma}|$ proportional to the deformation rate.

\section{B. Problem statement}

Coupling the above constitutive model with the mass and momentum conservation equations of the mixture, the problem can be formulated as a system of three equations for three unknowns: $\gamma_{e}$, the particle cage deformation; $\boldsymbol{u}$, the mixture velocity; and $p_{f}$, the pressure in the fluid phase:

$$
\begin{aligned}
\frac{\mathscr{D} \boldsymbol{\gamma}_{e}}{\mathscr{D} t}+\alpha|2 D(\boldsymbol{u})| \boldsymbol{\gamma}_{e}-2 D(\boldsymbol{u}) & =0 \\
\rho \frac{D \boldsymbol{u}}{D t}-\operatorname{div}\left\{-p_{f} \boldsymbol{I}+2 \eta D(\boldsymbol{u})+\alpha \eta_{e}|2 D(\boldsymbol{u})| \boldsymbol{\gamma}_{e}+\beta \eta_{e}\left(D(\boldsymbol{u}): \boldsymbol{\gamma}_{e}\right) \boldsymbol{\gamma}_{e}\right\} & =\rho \boldsymbol{g} \\
\operatorname{div} \boldsymbol{u} & =0
\end{aligned}
$$

The isochoric relation (6c) of the mixture is a consequence of mass conservation of the fluid and solid phases. From a mathematical point of view, the fluid pressure $p_{f}$ acts as a Lagrange multiplier associated to this isochoric constraint. The problem is closed by suitable initial and boundary conditions. In $(6 \mathrm{~b}), D / D t=\partial / \partial t+\boldsymbol{u} . \nabla$ denotes the Lagrange derivative and $\rho$ is the density of the mixture. From Hulsen [41], it is possible to show that the constitutive equation (6a) leads to a conformation tensor $\boldsymbol{b}_{e}=\boldsymbol{I}+\gamma_{e}$ that is always positive definite. Note that the volume fraction $\phi$, and hence $\rho$, are supposed to be constant during the flow: in agreement with experimental evidences [3], we assume the time scale for migration to be large compared to the typical time scales for microstructure evolution, and focus here on the short-time response of the suspensions. Otherwise, the system could be also coupled with an additional diffusion equation for the volume fraction [23]. 


\section{STATIONARY SHEAR FLOWS AND MICROSTRUCTURE ANISOTROPY}
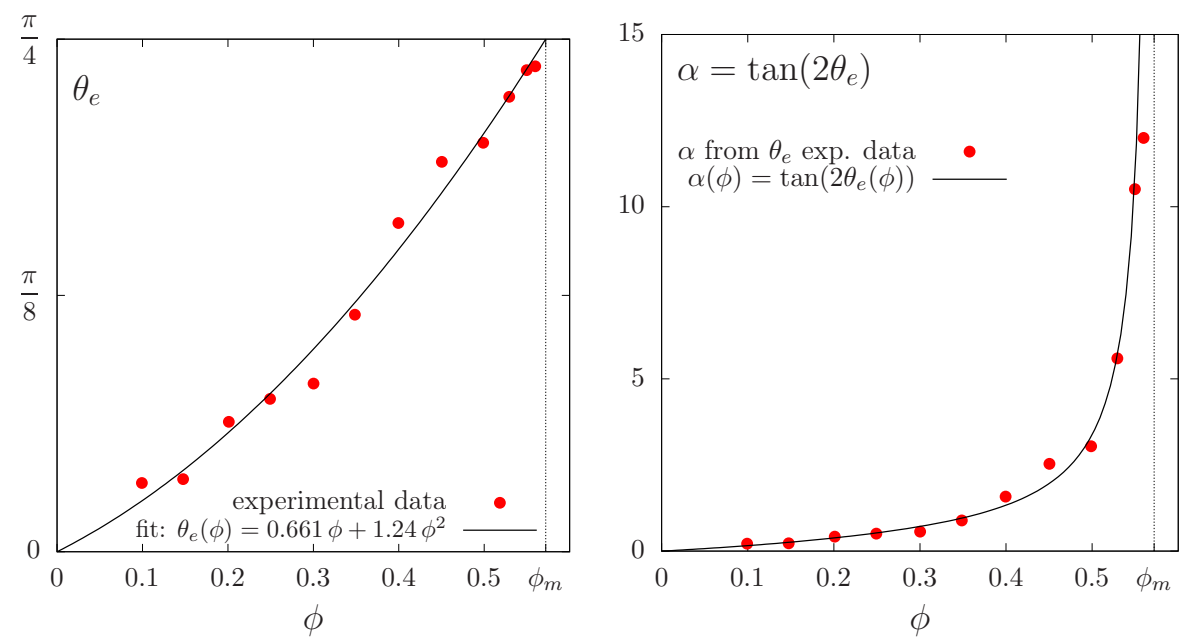

FIG. 2. (left) Depletion angle $\theta_{e}$ versus volume fraction $\phi$ : experimental data from [39], Fig. 5.11, and best fit with the second-order polynomial (10). (right) Dependence upon $\phi$ of model parameter $\alpha$.

Let us consider a simple shear flow. The $x$ axis is in the flow direction and the $y$ axis is in the direction of the gradient $\nabla=\left(0, \partial_{y}, 0\right)$. Let $\boldsymbol{u}(t, x, y, z)=\left(u_{x}(t, y), 0,0\right)$ be the velocity and $\dot{\gamma}(t)=\partial_{y} u_{x}$ be the spatially uniform shear rate. The constitutive equations (5a)-(5b) become, after expanding the upper-convected derivative [37] (chap. 4):

$$
\begin{array}{r}
\partial_{t} \gamma_{e, x y}+\alpha|\dot{\gamma}| \gamma_{e, x y}=\dot{\gamma} \\
\partial_{t} \gamma_{e, x x}-2 \dot{\gamma} \gamma_{e, x y}+\alpha|\dot{\gamma}| \gamma_{e, x x}=0 \\
\partial_{t} \gamma_{e, y y}+\alpha|\dot{\gamma}| \gamma_{e, y y}=0 \\
\partial_{t} \gamma_{e, z z}+\alpha|\dot{\gamma}| \gamma_{e, z z}=0 \\
\sigma_{x y}=\alpha \eta_{e}|\dot{\gamma}| \gamma_{e, x y}+\beta \eta_{e} \dot{\gamma} \gamma_{e, x y}^{2}+\eta \dot{\gamma} \\
\sigma_{x x}=\alpha \eta_{e}|\dot{\gamma}| \gamma_{e, x x}+\beta \eta_{e} \dot{\gamma} \gamma_{e, x y} \gamma_{e, x x}
\end{array}
$$

completed by an initial condition for $\gamma_{e}$. For simplicity, we assume an isotropic microstructure at $t=0$. Hence, $\gamma_{e, y y}(0)=\gamma_{e, z z}(0)=0$, which yields $\gamma_{e, y y}(t)=\gamma_{e, z z}(t)=0$ for all $t>0$. Similarly, $\gamma_{e, x z}(0)=\gamma_{e, y z}(0)=0$.

We first focus on a stationary simple shear flow where the shear rate is supposed to be constant and is denoted $\dot{\gamma}_{0}$. In that case, the solution writes explicitly:

$$
\begin{aligned}
\gamma_{e, x y} & =\operatorname{sgn}\left(\dot{\gamma}_{0}\right) \alpha^{-1}, \quad \gamma_{e, x x}=2 \alpha^{-2} \\
\sigma_{x y} & =\left(\eta+\left(1+\beta \alpha^{-2}\right) \eta_{e}\right) \dot{\gamma}_{0} \\
\sigma_{x x} & =2 \alpha^{-1}\left(1+\beta \alpha^{-2}\right) \eta_{e}\left|\dot{\gamma}_{0}\right|
\end{aligned}
$$

Observe that the model predicts a normal stress component proportional to the shear rate $\left|\dot{\gamma}_{0}\right|$. This feature is in agreement with several experimental observations [4]. In contrast, most classical viscoelastic models, such as Maxwell and Oldroyd models, predict normal stresses in shear flows proportional to $\dot{\gamma}_{0}^{2}$ [37] (p. 157). In the present model, normal stresses proportional to $\left|\dot{\gamma}_{0}\right|$ arise from the use in (3) of an "effective elastic modulus" that is itself proportional to $\left|\dot{\gamma}_{0}\right|$, as required to obtain a rate-independent rheological behavior. The particle pressure $p_{p}=-\operatorname{tr}(\boldsymbol{\tau}) / 3$ is given by, for the present stationary simple shear 
flow:

$$
p_{p}=-(2 / 3) \alpha^{-1}\left(1+\beta \alpha^{-2}\right) \eta_{e}\left|\dot{\gamma}_{0}\right|
$$

Thus, $p_{p}$ is also proportional to the shear rate $\left|\dot{\gamma}_{0}\right|$, again in agreement with experimental observations [42]. Finally note that, from (8b), the shear stress component $\sigma_{x y}$ also scales linearly with $\dot{\gamma}_{0}$, as expected.

Let us now turn to microstructural aspects, described by the particle cage deformation tensor $\gamma_{e}$. As we only consider stationary simple flows in this paragraph, we can assume without loss of generality that $\dot{\gamma}_{0}>0$. From (8a), the eigenvector associated to the largest eigenvalue of the tensor $\gamma_{e}$, makes an angle with the $x$ axis denoted as $\theta_{e}$ and given by:

$$
\begin{aligned}
\theta_{e} & =\operatorname{atan}\left(\frac{-1+\sqrt{1+\alpha^{2}}}{\alpha}\right)=\frac{1}{2} \operatorname{atan}(\alpha) \\
\Longleftrightarrow \alpha & =\tan \left(2 \theta_{e}\right)
\end{aligned}
$$

Since $\boldsymbol{b}_{e}=\boldsymbol{I}+\boldsymbol{\gamma}_{e}$, the tensors $\boldsymbol{b}_{e}$ and $\boldsymbol{\gamma}_{e}$ share the same eigensystem. The angle $\theta_{e}$ is thus also associated to largest eigenvalue of $\boldsymbol{b}_{e}$, i.e. to the dilation direction of the microstructure: in this direction, the probability to find two particles in contact is the smallest, and $\theta_{e}$ thus corresponds to the so-called depletion angle. Experimental data for the depletion angle $\theta_{e}$ versus volume fraction $\phi$ are presented by Blanc [39], Fig. 5.11, and are reproduced on Fig. 2 (left), together with a best fit using a second-order polynomial denoted by $\theta_{e}(\phi)$. Assuming $\theta_{e}(0)=0$ and $\theta_{e}\left(\phi_{m}\right)=\pi / 4$, with $\phi_{m}$ the maximum volume fraction of the suspension, the second-order polynomial template can be expressed as:

$$
\theta_{e}(\phi)=\delta_{e} \phi+\left(\frac{\pi}{4}-\delta_{e} \phi_{m}\right)\left(\frac{\phi}{\phi_{m}}\right)^{2}
$$

where $\phi_{m}=0.571$ and $\delta_{e}=0.661$ are adjusted through a nonlinear least square method, as implemented in gnuplot [43]. Through (9), the dependence upon $\phi$ of the $\alpha$ parameter of the present model is thus directly deduced from the experimental data (see Fig. 2, right):

$$
\alpha(\phi)=\tan \left(1.32 \phi+2.48 \phi^{2}\right)
$$

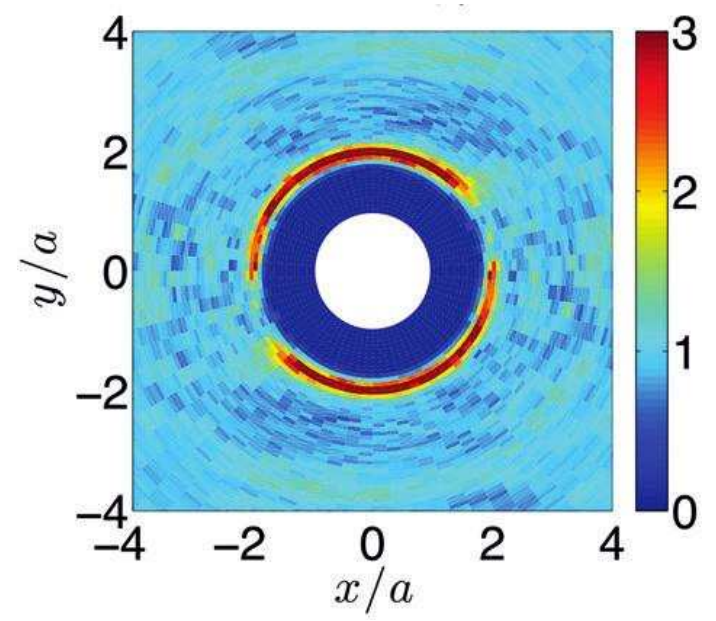

FIG. 3. Pair distribution function $g(x, y)$ : experimental observation by Blanc et al. [11] for a stationary shear flow with $\phi=0.35$.

In experiments or in numerical simulations, the microstructure of suspensions is generally represented through the pair distribution function $g(\boldsymbol{x})$. As an example, Fig. 3 shows, 
for a suspension at $\phi=0.35$ submitted to a a stationary shear flow, the experimentallydetermined evolution of $g(\boldsymbol{x})$ in the shear plane $(x, y)$ [11]. Recall that $g(\boldsymbol{x})$ is the conditional probability, when there is already a particle in $\boldsymbol{x}_{0}=0$, to find a particle at any location $\boldsymbol{x} \in \mathbb{R}^{2}$, normalized by the average particle density $\phi /\left(4 \pi a^{3} / 3\right)$ (where $a$ is particle radius). Observe on Fig. 3 that $g$ is zero in the central disk of diameter $2 a$, due to non-penetration of particles. It is maximum in a thin band $[2 a, 2 a+\delta]$, and then tends to 1 when the distance increases. Most of the relevant microstructure information is encoded in this thin band, whose thickness $\delta$ is sufficient to include contact and near-contact interactions, and which thus describes the average arrangement of neighboring particles $[9,10]$. Switching to polar coordinates $(r, \theta)$ in the shear plane, we assume, in order to simplify the modeling of $g$, that this function only depends upon $\theta$ in the thin band. We thus introduce its average value, denoted as $\tilde{g}(\theta)$, in radial direction in the thin band, and the associated probability distribution function $p(\theta)$ such that

$$
p(\theta)=\frac{\tilde{g}(\theta)}{\int_{-\pi}^{\pi} \tilde{g}(\theta) \mathrm{d} \theta}
$$

This distribution $p(\theta)$ is interpreted as the probability to find, for each particle in the suspension, a neighboring particle in the $\theta$ direction inside the thin band. Assuming furthermore that the probability to find a neighboring particle outside the thin band is negligible, $p(\theta)$ can be related to the fabric tensor $\langle\boldsymbol{n} \otimes \boldsymbol{n}\rangle$ introduced in section II A as follows:

$$
\langle\boldsymbol{n} \otimes \boldsymbol{n}\rangle=\int_{\theta=-\pi}^{\theta=\pi} \boldsymbol{n} \otimes \boldsymbol{n} p(\theta) \mathrm{d} \theta
$$

where $\boldsymbol{n}(\theta)=(\cos \theta, \sin \theta)$ is the unit outward normal vector to the unit circle. Observe that, as expected,

$$
\operatorname{tr}\langle\boldsymbol{n} \otimes \boldsymbol{n}\rangle=\int_{-\pi}^{\pi} p(\theta) \mathrm{d} \theta=1
$$

since $p$ is a probability distribution. Accordingly, from (1), we can thus postulate the following relation between $p(\theta)$ and the conformation tensor $\boldsymbol{b}_{e}$ introduced in our model:

$$
\int_{\theta=-\pi}^{\theta=\pi} \boldsymbol{n} \otimes \boldsymbol{n} p(\theta) \mathrm{d} \theta=\frac{\boldsymbol{b}_{e}^{-1}}{\operatorname{tr}\left(\boldsymbol{b}_{e}^{-1}\right)}
$$

As shown in appendix A, for a given conformation tensor $\boldsymbol{b}_{e},(13)$ can be used to reconstruct the probability distribution $p(\theta)$ through a Fourier mode decomposition.

From (13), the first Fourier mode of $p(\theta)$ can be expressed explicitly in terms of the parameter $\alpha$ and the depletion angle $\theta_{e}$ : see appendix A, relation (A3). This prediction is compared on Fig. 4 with experimental data from Blanc [39], Figs. 5.9 and 5.10. Observe that both predicted (in black) and experimental (in dotted-red) curves present two main lobes, separated by the depletion angle direction. The experimental probability distribution is however also affected by higher-frequency modes, which are are potentially very sensitive to both experimental errors from image preprocessing and the choice of the width of the thin band $[2 a, 2 a+\delta]$ used to integrate the pair distribution function, as pointed out by Blanc [39], Fig. 5.6. Fig. 5 represents the five first Fourier coefficients of the experimental data. Observe in general the rapid decrease of these coefficients, as expected. However, when the volume fraction $\phi$ becomes close to the maximal fraction $\phi_{m}$, the second mode dominates. A similar behavior has already been experimentally observed for dry granular material [44], and can be explained by steric exclusion of neighbors. This second Fourier mode cannot be determined by the present model, as explained in appendix A. 


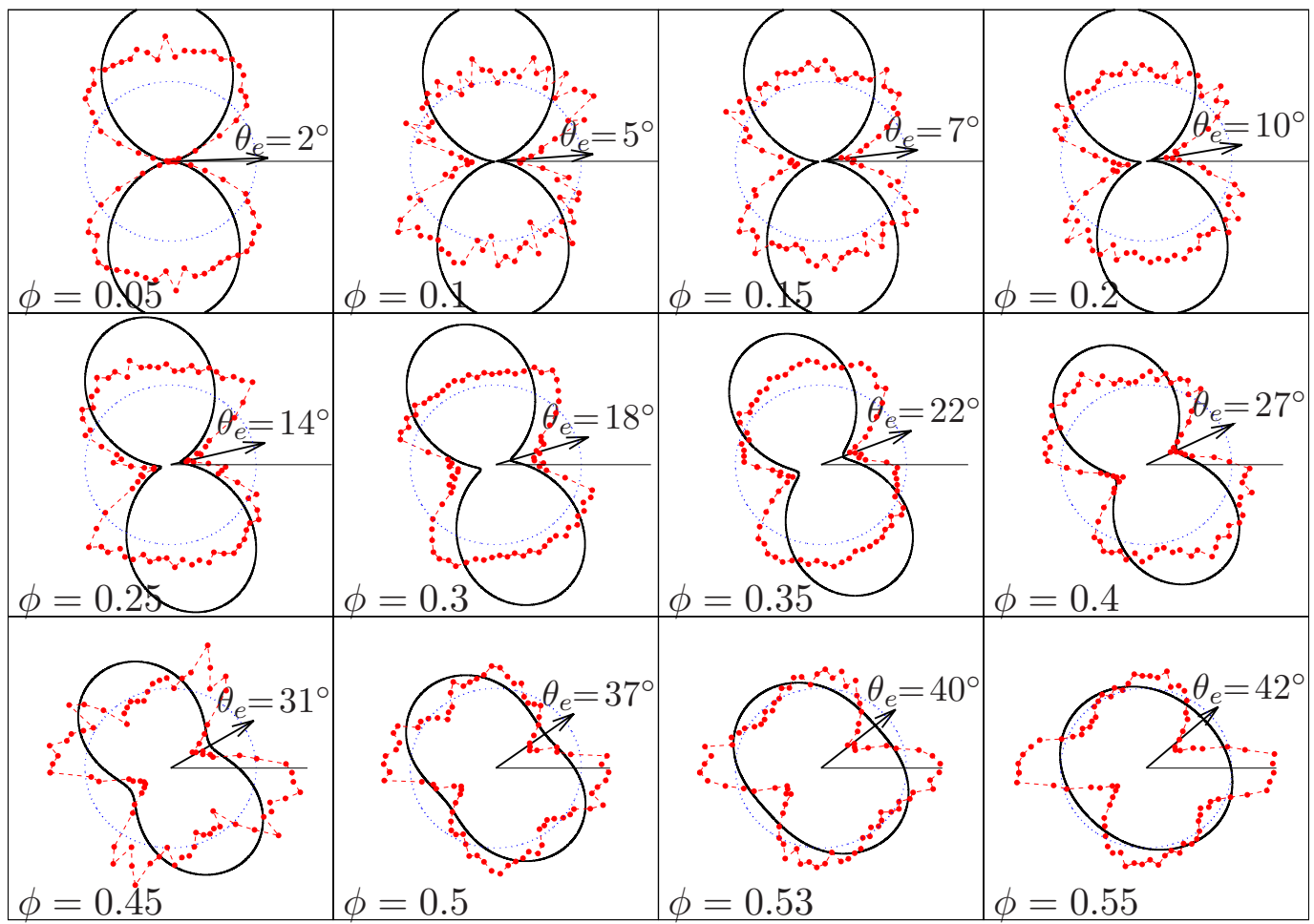

FIG. 4. Probability distribution function $p(\theta)$, represented in polar coordinates as $r=p(\theta)$. Comparison between model prediction (black curve) and experimental data (red dotted-curves) by Blanc [39], Fig. 5.10. The experimental pdfs are obtained as radial averages of the pair distribution function in the thin band corresponding to $r / a \in[1.87,2.14]$. The depletion angle is indicated by an arrow and its value is given in degrees. The dotted blue circle represents the equiprobable (isotropic) distribution $p(\theta)=1 /(2 \pi)$.

\section{TIME-DEPENDENT SIMPLE SHEAR FLOWS}

\section{A. Shear startup, reversal and pause}

For simple shear flows, the problem reduces to the time-dependent linear system of ordinary differential equation (7a)-(7f). For a constant applied shear rate $\dot{\gamma}_{0}$, the system can be explicitly solved by performing the change of variable $\gamma=\left|\dot{\gamma}_{0}\right| t$, where $\gamma$ represents the deformation. The solution writes:

$$
\begin{aligned}
& \gamma_{e, x y}(\gamma)=\left(1-e^{-\alpha \gamma}\right) \operatorname{sgn}\left(\dot{\gamma}_{0}\right) \alpha^{-1}+e^{-\alpha \gamma} \gamma_{e, x y}(0) \\
& \gamma_{e, x x}(\gamma)=\left(1-e^{-\alpha \gamma}\right) 2 \alpha^{-2}+e^{-\alpha \gamma}\left\{\gamma_{e, x x}(0)+2 \gamma\left(\operatorname{sgn}\left(\dot{\gamma}_{0}\right) \gamma_{e, x y}(0)-\alpha^{-1}\right)\right\}
\end{aligned}
$$

and, then, the total tress tensor $\boldsymbol{\sigma}$ is explicitly given by (7e)-(7f).

For a startup from a material at rest at $t=0$ with an isotropic microstructure, we have $\gamma_{e}(0)=0$. If a constant shear rate $\dot{\gamma}_{0}>0$ is imposed for $t>0$, the solution (14a)-(14b) becomes:

$$
\begin{aligned}
& \gamma_{e, x y}(\gamma)=\left(1-e^{-\alpha \gamma}\right) \alpha^{-1} \\
& \gamma_{e, x x}(\gamma)=\left(1-e^{-\alpha \gamma}\right) 2 \alpha^{-2}-e^{-\alpha \gamma} 2 \alpha^{-1} \gamma
\end{aligned}
$$

As shown on Fig. 6, this solution displays an exponential relaxation towards the steady state solution. Remark that the graph of the solution versus shear deformation $\gamma=\dot{\gamma}_{0} t$, 

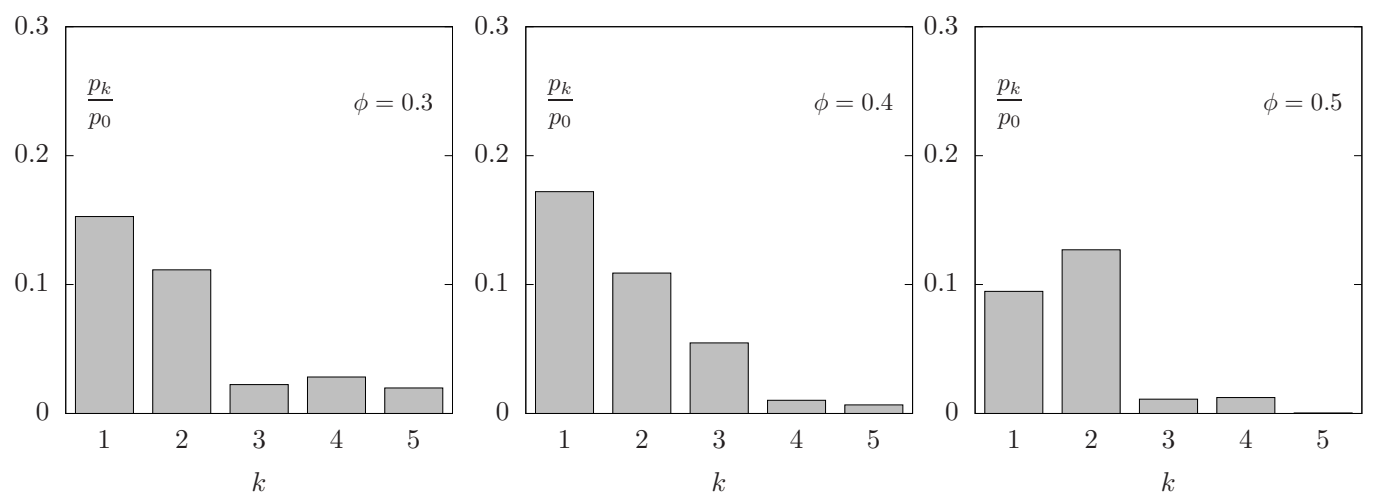

FIG. 5. Five first Fourier coefficients $p_{k}, k \geqslant 1$ of the probability distribution function $p(\theta)$, from experimental data by Blanc [39], Fig. 5.10, for $\phi=0.3,0.4$ and 0.5.

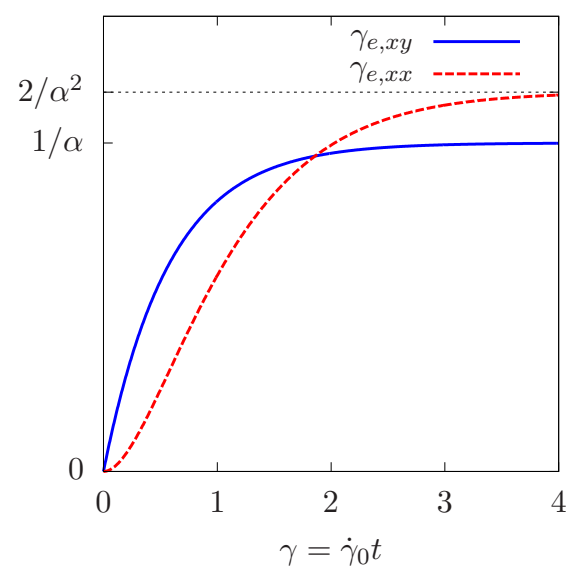

FIG. 6. Shear startup from rest: exponential relaxation of the $\gamma_{e}$ tensor components versus shear deformation $\gamma=\dot{\gamma}_{0} t$ (with $\alpha=\sqrt{3}$ ).

is invariant when changing the value of shear rate $\dot{\gamma}_{0}>0$. This constitutes a fundamental property of rate-independent materials.

Let us now turn to a case of shear reversal: the material is first sheared with a negative shear rate $-\dot{\gamma}_{0}$ until a first stationary regime is reached. Then, at $t=0$, the shear rate is suddenly reversed to the opposite value $+\dot{\gamma}_{0}>0$. In that case, $\gamma_{e, x y}(0)=-\alpha^{-1}, \gamma_{e, x x}(0)=2 \alpha^{-2}$ and the solution (14a)-(14b) becomes:

$$
\begin{aligned}
& \gamma_{e, x y}(\gamma)=\left(1-2 e^{-\alpha \gamma}\right) \alpha^{-1} \\
& \gamma_{e, x x}(\gamma)=\left(1-e^{-\alpha \gamma}\right) 2 \alpha^{-2}+e^{-\alpha \gamma}\left(2 \alpha^{-2}-4 \alpha^{-1} \gamma\right)
\end{aligned}
$$

As shown on Fig. 7 , at $t=0$, the particle cages, represented by the conformation tensor $\boldsymbol{b}_{e}=\boldsymbol{I}+\gamma_{e}$ as an ellipse, start to rotate towards a symmetrically opposite position. According to (9), the depletion angle increases from $\theta_{e}(0)=-\operatorname{atan}(\alpha) / 2$ at $t=0$ to reach asymptotically its new value $\theta_{e}(\infty)=+\operatorname{atan}(\alpha) / 2$. With the choice $\alpha=\sqrt{3}$ made in Fig. 7, we have $\theta_{e}(\infty)=\pi / 6$. The depletion angle is expressed at any $\gamma=\dot{\gamma}_{0} t$ from the $\gamma_{e}$ tensor components by:

$$
\theta_{e}(\gamma)=\operatorname{atan}\left(\frac{-\gamma_{e, x x}(\gamma)+\sqrt{\gamma_{e, x x}^{2}(\gamma)+4 \gamma_{e, x y}^{2}(\gamma)}}{\gamma_{e, x y}(\gamma)}\right)
$$




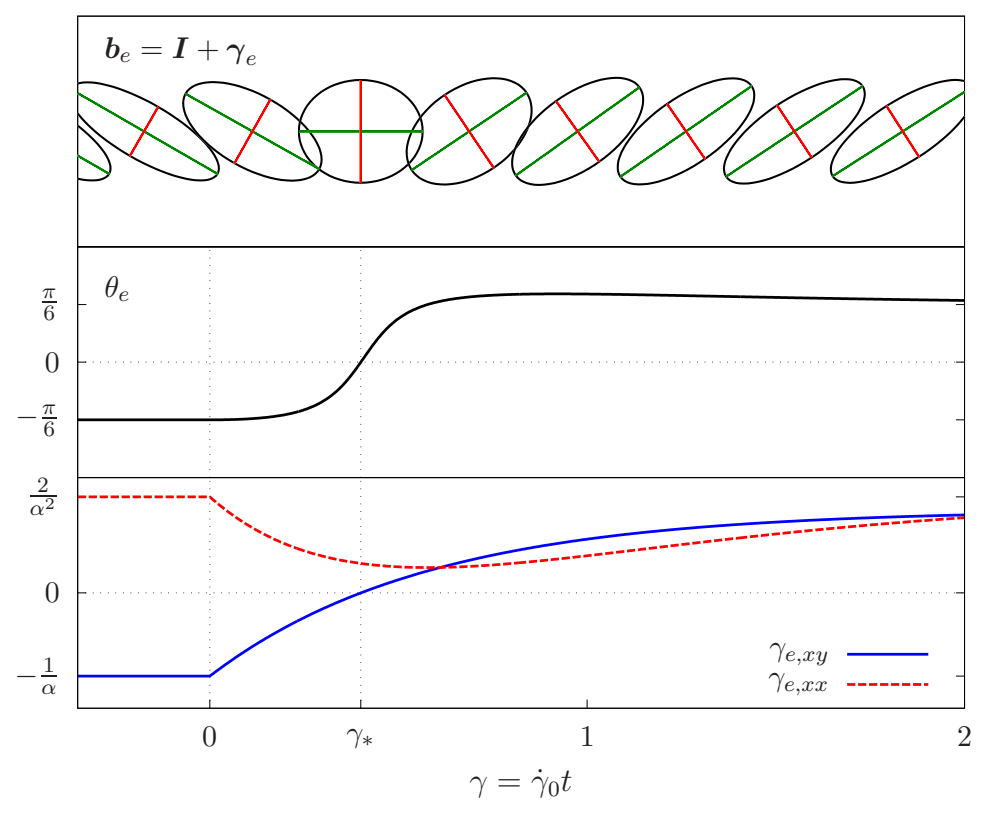

FIG. 7. Shear reversal at $t=0$ : evolution versus shear deformation $\gamma=\dot{\gamma}_{0} t$ of the conformation tensor $\boldsymbol{b}_{e}=\boldsymbol{I}+\boldsymbol{\gamma}_{e}$ represented as an ellipse. Eigenvector associated to compression (resp. dilation), i.e. to the smallest (resp. largest) eigenvalue of $\boldsymbol{b}_{e}$, is represented in red (resp. green). On the bottom, plots for the depletion angle $\theta_{e}(t)$, and the $\gamma_{e}$ tensor components versus shear deformation $\gamma=\dot{\gamma}_{0} t$. (Parameter $\alpha$ is taken as $\sqrt{3}$ in this plot.)

A Taylor development for small $\gamma_{e, x y}(\gamma)$ shows that $\theta_{e}(\gamma)$ vanishes when $\gamma_{e, x y}(\gamma)=0$, i.e. when the shear deformation is equal to the critical value $\gamma_{*}=\alpha^{-1} \log (2)$. For this critical deformation, the ellipse axis associated to the largest eigenvalue (in green on Fig. 7), is horizontal with $\gamma_{e, x y}\left(\gamma_{*}\right)=0$ and $\gamma_{e, x x}\left(\gamma_{*}\right)=2(1-\log (2)) \alpha^{-2}>0$. It means that the microstructure temporarily presents a fore-aft symmetry, but is not isotropic, i.e. $\boldsymbol{b}_{e} \neq \boldsymbol{I}$ when $\gamma=\gamma_{*}$. Fig. 7 also plots separately the two components $\gamma_{e, x y}$ and $\gamma_{e, x x}$ of the tensor: observe that $\operatorname{tr}\left(\gamma_{e}\right)=\gamma_{e, x x}$ is not constant during the reversal. This constitutes an important difference with approaches based on a fabric tensor $\langle\boldsymbol{n} \otimes \boldsymbol{n}\rangle$ that always has trace equal to one $[3,28,32,33,38]$.

For a general $\dot{\gamma}(t)$ evolution, the system of ordinary differential equations (7a)-(7f) is solved using lsode library [45], as interfaced in octave software [46]. Fig. 8 plots the response in stress components and depletion angle when applying a succession of startups and reversals, possibly separated by pauses. In agreement with experimental observations [7] (Fig. 3), when the imposed shear rate changes from $\dot{\gamma}_{0} \neq 0$ to zero, i.e. during a pause, both particle pressure $p_{p}=-\operatorname{tr}(\boldsymbol{\tau}) / 3=-\tau_{x x} / 3$ and shear stress $\sigma_{x y}$ instantaneously fall to zero. Observe however that the depletion angle remains constant during the pause: the microstructure is conserved. This latter feature can be deduced from constitutive equation (5a), which simply reduces to $\mathscr{D} \gamma_{e} / \mathscr{D} t=0$ when the shear rate is zero. After a pause, if the shear restarts suddenly in the same direction, experimental observations [7] (Fig. 3) showed that both particle pressure $p_{p}=-\tau_{x x} / 3$ and shear stress $\sigma_{x y}$ jump instantaneously to their previous stationary values. Conversely, if the shear rate restarts suddenly in the direction opposite to its previous value, e.g. $-\dot{\gamma}_{0}$, experimental observations by Narumi et al. [31], Fig. 3, showed that particle pressure $p_{p}$ progressively increases from zero to its previous stationary value while shear stress $\sigma_{x y}$ progressively decreases from zero to the opposite of its previous stationary value. As shown on Fig. 8, all these features are remarkably well captured by the present model. 


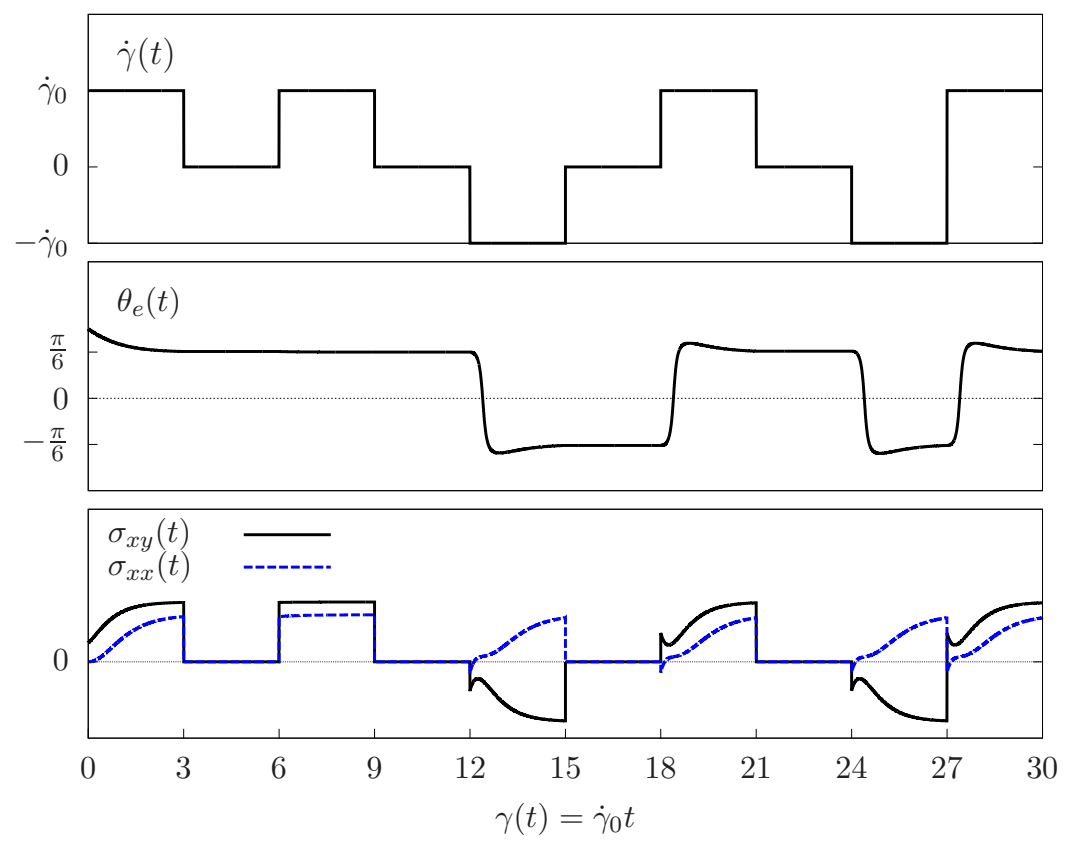

FIG. 8. Shear evolution with pauses, startups and reversals: evolution of imposed shear rate, depletion angle, and stress components versus shear deformation (choice of parameters: $\alpha=\sqrt{3}$, $\beta=5, \eta_{e}=10$ Pa.s, $\eta=10$ Pa.s).

The apparent viscosity of the suspension is defined as $\eta_{a p p}=\sigma_{x y} / \dot{\gamma}$. From (7e), we obtain:

$$
\eta_{a p p}(\gamma)=\eta+\eta_{e} \gamma_{e, x y}(\gamma)\left(\alpha+\beta \gamma_{e, x y}(\gamma)\right)
$$

Notice that the apparent viscosity is independent of the shear rate. Fig. 9 presents the evolution of apparent viscosity for a shear reversal, together with a sensitivity analysis to the model parameters. The apparent viscosity shows three regimes after the shear reversal: First, an instantaneous decrease is observed. The apparent viscosity then continues to decrease with a smooth shape until a minimum is reached. Finally, the apparent viscosity increases and relaxes exponentially to its stationary value. As shown in Fig. 9, these different regimes are diversely affected by the model parameters $\alpha, \beta, \eta_{e}$ and $\eta$. The parameter $\alpha$ controls the relaxation of the solution to its stationary value: the larger $\alpha$, the faster the solution reaches the stationary regime. In fact, $\alpha^{-1}$ interprets as a characteristic deformation for reaching the stationary regime. The parameter $\beta$ controls the existence of the smooth minimum and the shape of the curve around this minimum. When $\beta=0$, there is no smooth minimum, and the apparent viscosity is monotonically increasing immediately after the shear reversal. The viscosity $\eta_{e}$ influences the stationary plateau, while the minimum remains unchanged. Finally, the parameter $\eta$ globally shifts the apparent viscosity: note that this effect is obvious when considering (15).

\section{B. Comparison with experiments}

We quantitatively compared our model to the unsteady shear flow experiments of Blanc [39], sec 3.3 (see also $[8,11,47]$ ). This author performed shear reversal experiments in a Couette rheometer. The suspensions were prepared with polymethyl methacrylate (PMMA) spherical particles in a Newtonian oil at various volume fractions $\phi$ ranging from 0.30 to 0.50 . The experiments were performed at an imposed torque whose value was adjusted in order to obtain, for each volume fraction, similar angular velocities in the stationary regime. All 

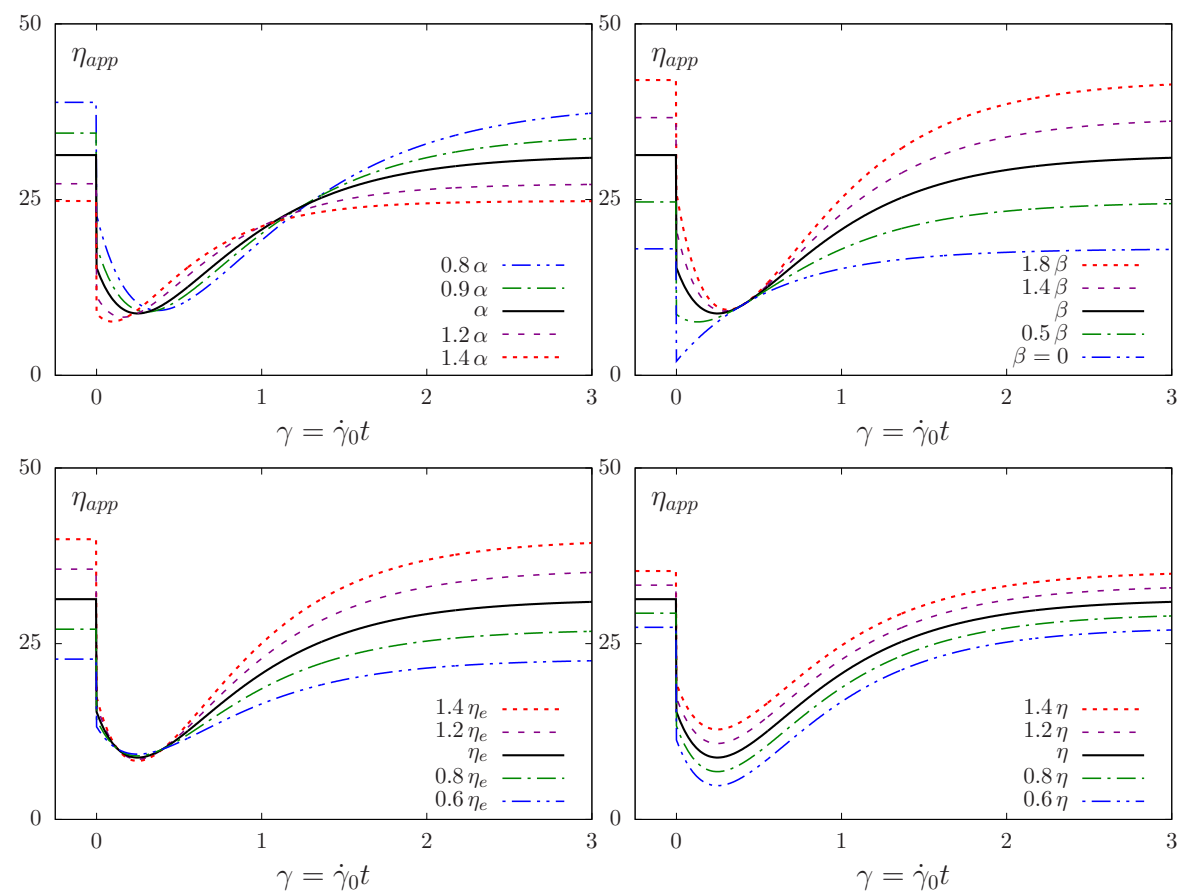

FIG. 9. Apparent viscosity during shear reversal: sensitivity of the response to the model parameters (base value of the parameters: $\alpha=\sqrt{3}, \beta=5, \eta_{e}=10$ Pa.s, $\eta=10$ Pa.s).

\begin{tabular}{|l|c|c|c|}
\hline description & unit & symbol & value \\
\hline \hline fluid viscosity & $P a . s$ & $\eta_{0}$ & 1.03 \\
\hline outer radius & $m$ & $R_{e}$ & $2.410^{-2}$ \\
\hline inner radius & $m$ & $R_{i}$ & $1.410^{-2}$ \\
\hline height & $m$ & $L$ & $4.510^{-2}$ \\
\hline \hline torque & $N . m$ & $T_{m}$ & imposed \\
\hline angular velocity & $s^{-1}$ & $\omega$ & measured \\
\hline shear stress & $P a$ & $\sigma_{x y}$ & $\frac{0.818 \times T_{m}}{2 \pi L R_{c}^{2}}$ \\
\hline shear rate & $s^{-1}$ & $\dot{\gamma}$ & $\frac{\omega R_{i}}{R_{e}-R_{i}}$ \\
\hline
\end{tabular}

TABLE I. Parameters of the shear reversal experiments of Blanc [39].

geometrical and material parameters of the experiments are summarized in Table I. Note that, experimentally, the suspensions have been found to be slightly shear-thinning, with a power index on the order 0.9 (see [39]). This slight shear-thinning is not considered in the following comparison with our model.

Neglecting the variations inside the gap, we assume the shear rate as uniform and consider this experiment as a simple shear flow. The problem is then described again by (7a)-(7f), where now $\sigma_{x y}$ is imposed and $\dot{\gamma}$ is unknown. Observe that, based on relation (7e), the unknown shear rate $\dot{\gamma}$ expresses explicitly in terms of the unknown $\gamma_{e, x y}$ and the given data $\sigma_{x y}$ (see appendix B). This expression can then be inserted in (7a), yielding a nonlinear scalar ordinary differential equation for $\gamma_{e, x y}$. This equation does not admit, to our knowledge, an explicit solution and should be solved numerically. As in the previous paragraph, the numerical procedure uses lsode library [45]. 

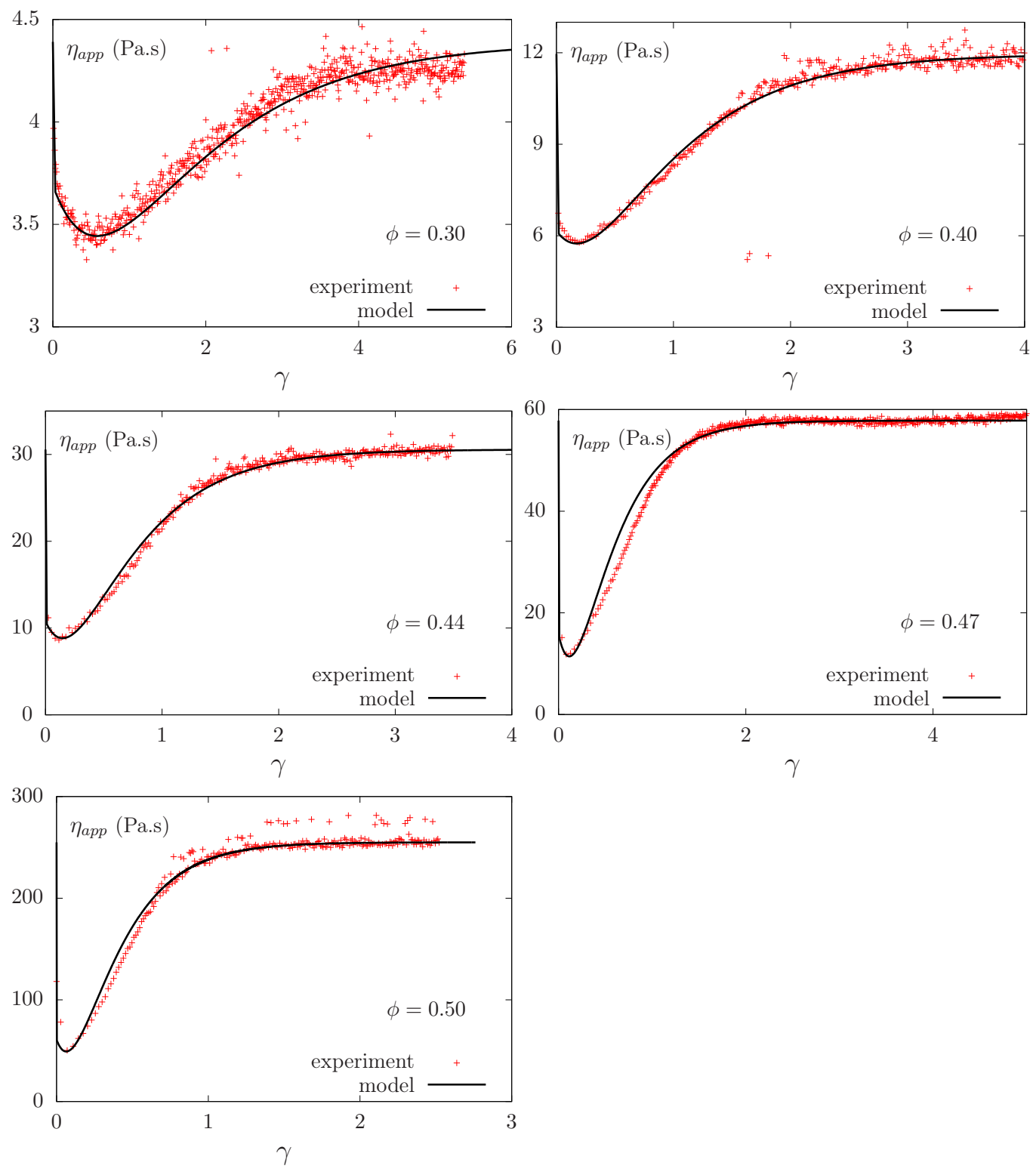

FIG. 10. Shear reversal: apparent viscosity $\eta_{a p p}$ vs deformation $\gamma$. Comparison between experimental measurements from Blanc [39] for a suspension of PMMA particles in a Couette geometry and computations with the present model. For each volume fraction $\phi$, the three model parameters $\beta, \eta_{e}$, and $\eta$ were obtained through a best-fit procedure. Parameter values are indicated in Table II.

The present model involves four parameters that need to be determined: $\alpha, \beta, \eta_{e}$ and $\eta$. The $\alpha$ parameter has been already identified for this experimental setup, and its dependence upon $\phi$ is given by (11). For each volume fraction, identification of the three other parameters can be performed based on the evolution of the apparent viscosity $\eta_{a p p}=\sigma_{x y} / \dot{\gamma}$ during the shear reversals, as illustrated in the previous sensitivity analysis.

Fig. 10 presents direct comparisons between model prediction and experimental measurements of the apparent viscosity. Observe that the sudden decrease of the apparent viscosity the after shear reversal, and its relaxation to the stationary value, are qualitatively and quantitatively very well reproduced by the present continuous model, and this for the dif- 


\begin{tabular}{|l|l||l|l|l|l|}
\hline$\phi$ & $T_{m}\left(10^{-3} \mathrm{Nm}\right)$ & $\alpha$ & $\beta$ & $\eta_{e}(\mathrm{~Pa} . \mathrm{s})$ & $\eta(\mathrm{Pa} . \mathrm{s})$ \\
\hline \hline 0.30 & 0.07 & 0.715 & 0.79 & 0.35 & 3.5 \\
\hline 0.40 & 0.10 & 1.33 & 1.5 & 2.9 & 6.6 \\
\hline 0.44 & 0.25 & 1.80 & 3.0 & 9.9 & 11.5 \\
\hline 0.47 & 0.50 & 2.37 & 5.3 & 21 & 17 \\
\hline 0.50 & 2.00 & 3.38 & 9.3 & 97 & 79 \\
\hline
\end{tabular}

TABLE II. Model parameters used to fit the experiments of Blanc [39].

ferent volume fractions investigated. For $\phi=0.47$, the apparent viscosity measured during the experiments displays a very slowly increasing trend for large deformations $\gamma$. This feature, which is obviously not captured by the model, could be due to slow migration of the particles induced by the small variations of the shear rate in the Couette gap.
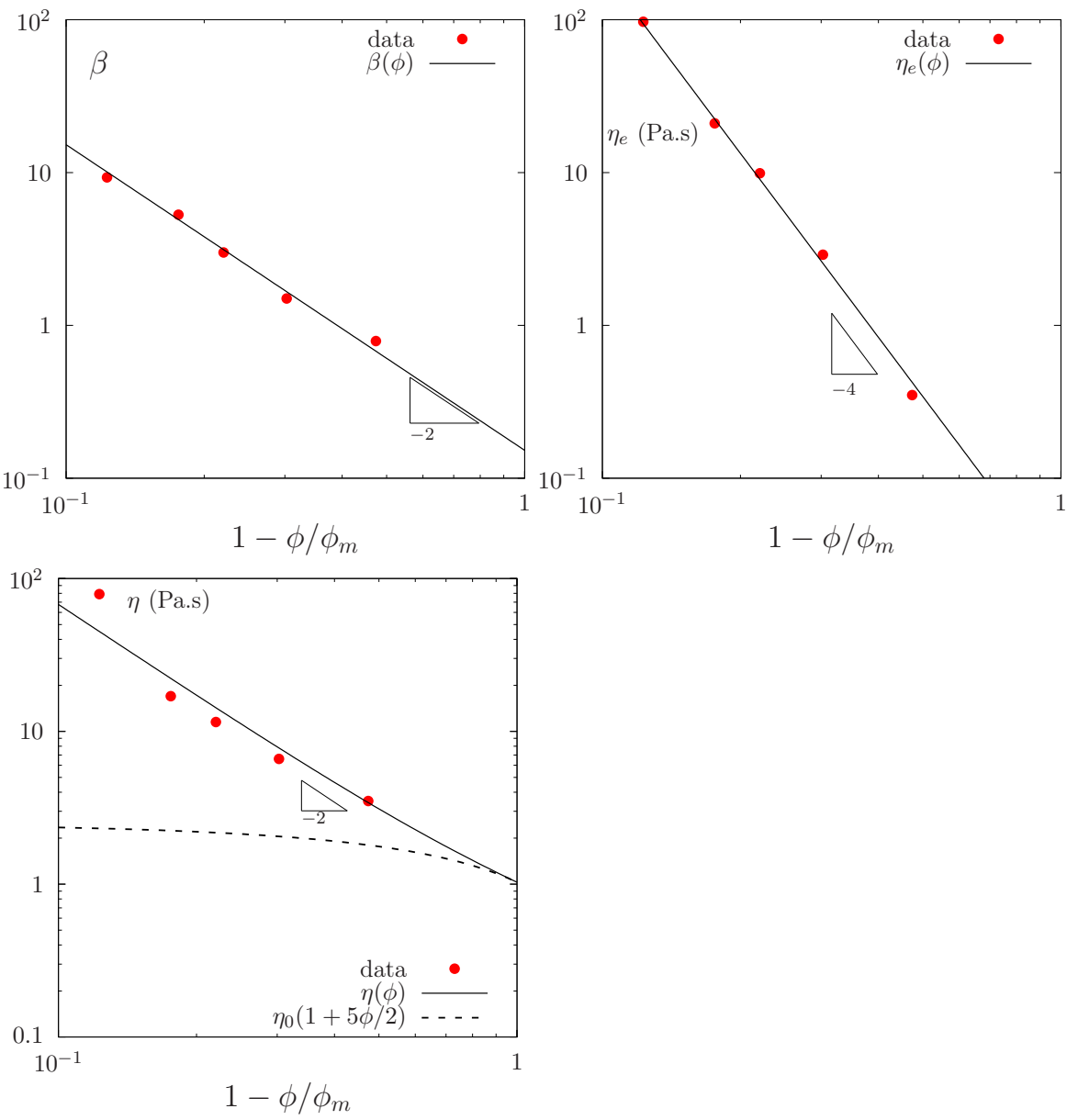

FIG. 11. Evolution with $\phi$ of the model parameters $\beta, \eta_{e}$, and $\eta$, and best fits provided by expressions (16a)-(16c).

Table II summarizes, for each volume fraction, the values of the four adjustable parameters $\alpha, \beta, \eta_{e}$ and $\eta$ provided by the fitting. Fig. 11 shows the dependency upon $\phi$ of the parameters $\beta, \eta_{e}$ and $\eta$. The regularity of these dependencies suggests the existence of 


\begin{tabular}{|c|c|c|c|c|}
\hline$\phi_{m}$ & $\delta_{e}(\mathrm{rad})$ & $\bar{\beta}$ & $\bar{\eta}_{e}(\mathrm{~Pa} . \mathrm{s})$ & $\omega$ \\
\hline \hline 0.571 & 0.661 & 0.152 & 0.0213 & 0.652 \\
\hline
\end{tabular}

TABLE III. Fitting parameters involved in expressions (10) and (16a)-(16c).

material functions with the following forms:

$$
\begin{aligned}
\beta(\phi) & =\bar{\beta}\left(1-\frac{\phi}{\phi_{m}}\right)^{-2} \\
\eta_{e}(\phi) & =\bar{\eta}_{e}\left(1-\frac{\phi}{\phi_{m}}\right)^{-4} \\
\eta(\phi) & =\eta_{0}\left(1-\omega+\left(\frac{5}{2}-\frac{2 \omega}{\phi_{m}}\right) \phi+\omega\left(1-\frac{\phi}{\phi_{m}}\right)^{-2}\right)
\end{aligned}
$$

Hence, $\beta$ and $\eta_{e}$ vs $\phi$ are expressed by simple power-law dependencies diverging at $\phi=\phi_{m}$, where $\phi_{m}$ is the maximum volume fraction of the suspension. Expression (16c) for $\eta(\phi)$ is an original extension of Krieger and Dougherty [48]'s rule, associated to the -2 power-law index, where $\omega$ is a balance parameter. Note that, when volume fraction is small, the first order development of (16c) coincides with Einstein [17]'s rule $\eta(\phi) / \eta_{0}=1+5 \phi / 2+\mathscr{O}\left(\phi^{2}\right)$ for any value of $\phi_{m}$ and $\omega \in[0,1]$. Best-fitted values of all the material parameters involved in (16a)-(16c) are indicated in Table III. Lastly, recall that the evolution of $\alpha$ upon $\phi$ was obtained independently, and is given by (11).

\section{v. DISCUSSION AND CONCLUSIONS}

This paper proposes a minimal tensorial model attempting to clearly represent the role of microstructure on the apparent viscosity of non-colloidal suspensions of rigid particles. The contribution to the total stress of the suspension of local anisotropic particle arrangements, is accounted for through a specific microstructure stress. This microstructure stress is expressed as a function of a local conformation tensor, whose evolution is governed by a rate-independent viscoelastic-like differential equation. Qualitatively, this model proves capable of reproducing several important non-Newtonian trends exhibited by concentrated suspensions. First, the development of an anisotropic, and fore-aft asymmetric, microstructure in simple shear is well captured by the conformation tensor. As expected, the stationary microstructure is independent of shear rate (see (9)). The depletion angle, which corresponds to the largest eigenvalue of the the conformation tensor, is a function of a single model parameter $\alpha$ that can be adjusted to fit experimental observations. Second, in time-dependent cases, the model predicts transient responses associated to the progressive relaxation of the microstructure towards its stationary state. In agreement with experimental observations, these transient responses occur for shear reversals (due to the associated reversal of anisotropy direction), but not for changes of shear rate with the same sign (since microstructure is rate-independent). Also in agreement with experiments, the microstructure remains frozen during shear pauses, and its evolution during the transients is fully controlled by the shear deformation. The critical deformation to reach the stationary regime is directly related, again, to the parameter $\alpha$.

Overall, the model presented here includes only 4 constitutive parameters. Besides $\alpha$, two viscosities $\eta$ and $\eta_{e}$ represent the base viscosity of the suspension for an isotropic microstructure and the excess viscosity induced by microstructure anisotropy, respectively, while the non-linearity parameter $\beta$ controls the early stage of the transients. This limited number of parameters, and their clear physical meaning, is an advantage compared to most previous microstructure-based rheological models proposed in the literature $[28,32,34]$. In particular, parameter identification for quantitative comparisons with experimental data is 
relatively straightforward. We showed that the model is capable of quantitatively reproducing the complex transient evolution of apparent viscosity observed after shear reversals for a large range of volume fractions. Both the immediate response, characterized by an instantaneous drop followed by a smooth minimum, and the subsequent exponential relaxation, are well captured. Note that the quadratic term in (5b), and the parameter $\beta$, are essential to obtain the smooth minimum observed in experimental data. To our knowledge, it is the first time that a microstructure-based rheological model is successfully compared to such a wide experimental data set. This comparison allowed us to derive material functions for the evolution of the constitutive parameters with volume fraction. Noteworthily, the values of the parameter $\alpha$ were determined from microstructure data (depletion angle), and then applied without adjustment to model the transient response. This validates the use of a single parameter controlling both microstructure anisotropy and the characteristic deformation during transients.

As a further quantitative validation, the model also proved capable of reproducing not only the depletion angle, but the overall shape of the pair distribution function. Here also, it is the first time, to our knowledge, that a continuous model is used to obtain detailed microstructural predictions in agreement with experimental data. Accounting for higherfrequency modes would further improve the prediction of the pair distribution function both for high and low values of the volume fraction, but would require the consideration of higherorder structure tensors in the model. Other promising prospects include the addition of a friction term to the microstructure stress, which could prove important for modeling volume fractions close to $\phi_{m}$ and/or experiments performed at imposed particle pressure [49].

Future works shall also consider in more details the issue of normal stresses. Indeed, another important non-Newtonian rheological feature exhibited by non-colloidal suspensions is the development of normal stress differences in simple shear flow, with negative values of $N_{2}$, an ongoing debate concerning the sign of $N_{1}$, and a ratio $\left|N_{2} / N_{1}\right|$ on the order of three, typically [4, 50-53]. In agreement with experimental observations, our model effectively predicts that microstructure anisotropy is associated to the existence of normal stresses proportional to shear rate. However, expressions of stresses in simple shear lead to $N_{2}=0$ and $N_{1}>0$ (see $\left.(8 \mathrm{c})\right)$. As a consequence, the particle pressure $p_{p}$, expressed in $(8 \mathrm{~d})$, has a sign opposite to that expected. This indicates that, although the minimal model presented here is capable to reproduce microstructure evolutions, additional degrees of freedom would be needed to capture the full rheological behavior of suspensions. These improvements will be required to consider, e.g., more complex non-viscosimetric flows such as extensional flows [54] or flows around an obstacle [55, 56]. These improvements are also required in order to predict particle migration, by considering the microstructure stress $\tau$ as the driver of the particle flux, through an approach analogous to SBM [16].

\section{ACKNOWLEDGMENTS}

It is a pleasure to thank Frédéric Blanc, Élisabeth Lemaire, Laurent Lobry and François Peters for fruitful discussions about the rheology of suspensions and for providing us data files of experimental measurements used in this paper for comparison with our model. The authors would like to thank the three anonymous reviewers for their careful reading of the paper and their constructive comments.

\section{REFERENCES}

[1] Stickel, J. J. and Powell, R. L., "Fluid mechanics and rheology of dense suspensions," Ann. Rev. Fluid Mech. 37, 129-149 (2005).

[2] Guazzelli, E. and Morris, J. F., A physical introduction to suspension dynamics, (Cambridge University Press, UK2012). 
[3] Denn, M. M. and Morris, J. F., "Rheology of non-brownian suspensions," Ann. Rev. Chem. Biomol. Eng. 5, 203-228 (2014).

[4] Zarraga, I. E., Hill, D. A., and Leighton Jr, D. T., "The characterization of the total stress of concentrated suspensions of noncolloidal spheres in Newtonian fluids," J. Rheol. 44, 185-220 (2000).

[5] Ovarlez, G., Bertrand, F., and Rodts, S., "Local determination of the constitutive law of a dense suspension of noncolloidal particles through magnetic resonance imaging," J. Rheol. 50, 259-292 (2006).

[6] Gadala-Maria, F. and Acrivos, A., "Shear-induced structure in a concentrated suspension of solid spheres," J. Rheol. 24, 799-814 (1980).

[7] Kolli, V. G., Pollauf, E. J., and Gadala-Maria, F., "Transient normal stress response in a concentrated suspension of spherical particles," J. Rheol. 46, 321-334 (2002).

[8] Blanc, F., Peters, F., and Lemaire, E., "Local transient rheological behavior of concentrated suspensions," J. Rheol. 55, 835-854 (2011).

[9] Brady, J. F. and Morris, J. F., "Microstructure of strongly sheared suspensions and its impact on rheology and diffusion," J. Fluid Mech. 348, 103-139 (1997).

[10] Morris, J. F., "A review of microstructure in concentrated suspensions and its implications for rheology and bulk flow," Rheol. Acta 48, 909-923 (2009).

[11] Blanc, F., Lemaire, E., Meunier, A., and Peters, F., "Microstructure in sheared non-Brownian concentrated suspensions," J. Rheol. 57, 273-292 (2013).

[12] Drazer, G., Koplik, J., Khusid, B., and Acrivos, A., "Deterministic and stochastic behaviour of nonBrownian spheres in sheared suspensions," J. Fluid Mech. 460, 307-335 (2002).

[13] Metzger, B. and Butler, J. E., "Irreversibility and chaos: role of long-range hydrodynamic interactions in sheared suspensions," Phys. Rev. E 82, 051406 (2010).

[14] Gallier, S., Lemaire, E., Peters, F., and Lobry, L., "Rheology of sheared suspensions of rough frictional particles," J. Fluid Mech. 757, 514-549 (2014).

[15] Morris, J. F. and Boulay, F., "Curvilinear flows of noncolloidal suspensions: the role of normal stresses," J. Rheol. 43, 1213-1237 (1999).

[16] Miller, R. M., Singh, J. P., and Morris, J. F., "Suspension flow modeling for general geometries," Chem. Eng. Sci. 64, 4597-4610 (2009).

[17] Einstein, A., "Eine neue bestimmung der moleküldimensionen," Ann. Phys. ser. 4 19, $289-306$ (1906).

[18] Einstein, A., Investigation on the theory of the Brownian movement, (Dover, Mineola, NY, USA1956).

[19] Jackson, R., "Locally averaged equations of motion for a mixture of identical spherical particles and a Newtonian fluid," Chem. Eng. Sci. 52, 2457-2469 (1997).

[20] Jackson, R., The dynamics of fluidized particles, (Cambridge University Press, UK2000).

[21] Nott, P. R., Guazzelli, E., and Pouliquen, O., "The suspension balance model revisited," Phys. Fluids 23, 043304 (2011).

[22] Nott, P. R. and Brady, J. F., "Pressure-driven flow of suspensions: simulation and theory," J. Fluid Mech. 275, 157-199 (1994).

[23] Miller, R. M. and Morris, J. F., "Normal stress-driven migration and axial development in pressuredriven flow of concentrated suspensions," J. Non-Newt. Fluid Mech. 135, 149-165 (2006).

[24] Lhuillier, D., "Migration of rigid particles in non-Brownian viscous suspensions," Phys. Fluids 21, 023302 (2009).

[25] Lipscomb, G. G., Denn, M. M., Hur, D. U., and Boger, D. V., "The flow of fiber suspensions in complex geometries," J. Non-Newt. Fluid Mech. 26, 297-325 (1988).

[26] Reddy, B. D. and Mitchell, G. P., "Finite element analysis of fibre suspension flows," Comput. Meth. Appl. Mech. Eng. 190, 2349-2367 (2001).

[27] Hand, G. L., "A theory of anisotropic fluids," J. Fluid Mech. 13, 33-46 (1962).

[28] Goddard, J. D., "A dissipative anisotropic fluid model for non-colloidal particle dispersions," J. Fluid Mech. 568, 1-17 (2006).

[29] Kolymbas, D., "An outline of hypoplasticity," Arch. Appl. Mech. 61, 143-151 (1991).

[30] Phan-Thien, N., "Constitutive equation for concentrated suspensions in Newtonian liquids," J. Rheol. 39, 679-695 (1995). 
[31] Narumi, T., See, H., Honma, Y., Hasegawa, T., Takahashi, T., and Phan-Thien, N., "Transient response of concentrated suspensions after shear reversal," J. Rheol. 46, 295-305 (2002).

[32] Phan-Thien, N., Fan, X.-J., and Khoo, B. C., "A new constitutive model for monodispersed suspensions of spheres at high concentrations," Rheol. Acta 38, 297-304 (1999).

[33] Phan-Thien, N., Fan, X.-J., and Zheng, R., "A numerical simulation of suspension flow using a constitutive model based on anisotropic interparticle interactions," Rheol. Acta 39, 122-130 (2000).

[34] Stickel, J. J., Phillips, R. J., and Powell, R. L., "A constitutive model for microstructure and total stress in particulate suspensions," J. Rheol. 50, 379-413 (2006).

[35] Stickel, J. J., Phillips, R. J., and Powell, R. L., "Application of a constitutive model for particulate suspensions: time-dependent viscometric flows," J. Rheol. 51, 1271-1302 (2007).

[36] Yapici, K., Powell, R. L., and Phillips, R. J., "Particle migration and suspension structure in steady and oscillatory plane Poiseuille flow," Phys. Fluids 21, 053302 (2009).

[37] Saramito, P., Complex fluids: modelling and algorithms, (Springer2016).

[38] Chacko, R. N., Mari, R., Fielding, S. M., and Cates, M. E., "Shear reversal in dense suspensions: the challenge to fabric evolution models from simulation data," submitted (2017).

[39] Blanc, F., Rhéologie et microstructure des suspensions concentrées non browniennes, Ph.D. thesis, Université Nice Sophia Antipolis (2011).

[40] Oldroyd, J. G., "On the formulation of rheological equations of states," Proc. R. Soc. Lond. A 200, 523-541 (1950).

[41] Hulsen, M. A., "A sufficient condition for a positive definite configuration tensor in differential models," J. Non-Newt. Fluid Mechanics 38, 93-100 (1990).

[42] Deboeuf, A., Gauthier, G., Martin, J., Yurkovetsky, Y., and Morris, J. F., "Particle pressure in a sheared suspension: a bridge from osmosis to granular dilatancy," Phys. Rev. Lett. 102, 108301 (2009).

[43] Williams, T. and Keley, C., "gnuplot: an interactive program," (2010), http://www.gnuplot.info.

[44] Troadec, H., Radjai, F., Roux, S., and Charmet, J. C., "Model for granular texture with steric exclusion," Phys. Rev. E 66, 041305 (2002).

[45] Radhakrishnan, K. and Hindmarsh, A. C., "Description and use of LSODE, the Livermore solver for ordinary differential equations," Technical Report UCRL-ID-113855, LLNL (1993).

[46] Eaton, J. W., Bateman, D., and Hauberg, S., Octave: A high-level interactive language for numerical computations, (Free software fundation2011), http://www.gnu.org/software/octave.

[47] Blanc, F., Peters, F., and Lemaire, E., "Experimental signature of the pair trajectories of rough spheres in the shear-induced microstructure in noncolloidal suspensions," Phys. Rev. Lett. 107, 208302 (2011).

[48] Krieger, I. M. and Dougherty, T. J., "A mechanism for non-newtonian flow in suspensions of rigid spheres," Trans. Soc. Rheol. 3, 137-152 (1959).

[49] Boyer, F., Guazzelli, É., and Pouliquen, O., "Unifying suspension and granular rheology," Phys. Rev. Lett. 107, 188301 (2011).

[50] Boyer, F., Pouliquen, O., and Guazzelli, É., "Dense suspensions in rotating-rod flows: normal stresses and particle migration," J. Fluid Mech. 686, 5-25 (2011).

[51] Couturier, É., Boyer, F., Pouliquen, O., and Guazzelli, É., "Suspensions in a tilted trough: second normal stress difference," J. Fluid Mech. 686, 26-39 (2011).

[52] Dai, S.-C., Bertevas, E., Qi, F., and Tanner, R. I., "Viscometric functions for noncolloidal sphere suspensions with Newtonian matrices," J. Rheol. 57, 493-510 (2013).

[53] Dbouk, T., Lobry, L., and Lemaire, E., "Normal stresses in concentrated non-Brownian suspensions," J. Fluid Mech. 715, 239-272 (2013).

[54] Dai, S. and Tanner, R. I., "Elongational flows of some non-colloidal suspensions," Rheol. Acta 56, 63-71 (2017).

[55] Haddadi, H., Shojaei-Zadeh, S., Connington, K., and Morris, J. F., "Suspension flow past a cylinder: particle interactions with recirculating wakes," J. Fluid Mech. 760, R2 (2014).

[56] Dbouk, T., "A suspension balance direct-forcing immersed boundary model for wet granular flows over obstacles," J. Non-Newt. Fluid Mech. 230, 68-79 (2016). 


\section{Appendix A: Computation of the probability distribution function}

Let $\mu_{ \pm}$be the two eigenvalues of the fabric tensor $\langle\boldsymbol{n} \otimes \boldsymbol{n}\rangle$, with $\mu_{-} \leqslant \mu_{+}$and $\boldsymbol{e}_{-}=\left(\cos \left(\theta_{e}\right), \sin \left(\theta_{e}\right)\right)$ and $\boldsymbol{e}_{+}=\left(-\sin \left(\theta_{e}\right), \cos \left(\theta_{e}\right)\right)$ the two corresponding eigenvectors, where $\theta_{e}$ is the depletion angle. Expressing (12) in the eigenbasis, observing that $\boldsymbol{n} . \boldsymbol{e}_{-}=\cos \left(\theta-\theta_{e}\right)$ and $\boldsymbol{n} . \boldsymbol{e}_{+}=\sin \left(\theta-\theta_{e}\right)$, we get:

$$
\begin{aligned}
& \int_{-\pi}^{\pi} \cos ^{2}\left(\theta-\theta_{e}\right) p(\theta) \mathrm{d} \theta=\mu_{-} \\
& \int_{-\pi}^{\pi} \sin ^{2}\left(\theta-\theta_{e}\right) p(\theta) \mathrm{d} \theta=\mu_{+}
\end{aligned}
$$

Note that, by construction, $p(\theta)$ is even (see Fig. 3). Then, expressing $p(\theta)$ in terms of a Fourier series as:

$$
p(\theta)=\sum_{k \geqslant 0} p_{k} \cos \left(2 k\left(\theta-\theta_{e}\right)\right)
$$

where $p_{k} \in \mathbb{R}, k \geqslant 0$ are the Fourier coefficients, we obtain from (A1a)-(A1b), after computation of the integrals, that $p_{0}=1 /(2 \pi)$ and $p_{1}=-\left(\mu_{+}-\mu_{-}\right) /(2 \pi)$. The coefficients $p_{k}$ for $k \geqslant 2$ remain undetermined. Observe from Fig. 5 that, in experimental data, these coefficients present a fast decrease. By retaining only the two first coefficients, the present model is able to predict the following probability distribution:

$$
p(\theta)=\frac{1}{2 \pi}\left(1-\left(\mu_{+}-\mu_{-}\right) \cos \left\{2\left(\theta-\theta_{e}\right)\right\}\right)
$$

Note that such expression was previously used by Troadec et al. [44], eqn (1). Remark that $\theta_{e}$ minimizes $p(\theta)$ : as expected, the depletion angle is the direction where the probability to find a neighbor particle is minimal.

In the present model, the fabric tensor is expressed from (13) by $\langle\boldsymbol{n} \otimes \boldsymbol{n}\rangle=\boldsymbol{b}_{e}^{-1} / \operatorname{tr}\left(\boldsymbol{b}_{e}^{-1}\right)$ with $\boldsymbol{b}_{e}=\boldsymbol{I}+\gamma_{e}$. Accordingly, the two eigenvalues of the fabric tensor $\langle\boldsymbol{n} \otimes \boldsymbol{n}\rangle$ are

$$
\mu_{+}=\frac{\left(1+\lambda_{-}\right)^{-1}}{\left(\lambda_{+}+1\right)^{-1}+\left(\lambda_{-}+1\right)^{-1}} \quad \text { and } \quad \mu_{-}=\frac{\left(1+\lambda_{+}\right)^{-1}}{\left(\lambda_{+}+1\right)^{-1}+\left(\lambda_{-}+1\right)^{-1}}
$$

where $\lambda_{ \pm}$denotes the two eigenvalues of $\gamma_{e}$. From (8a), we have $\lambda_{ \pm}=\left(1 \pm \sqrt{1+\alpha^{2}}\right) / \alpha^{2}$. Then $\mu_{+}-\mu_{-}=1 / \sqrt{1+\alpha^{2}}$ and the previous relation (A2) writes explicitly in terms of the model parameter $\alpha$ only:

$$
p(\theta)=\frac{1}{2 \pi}\left(1-\frac{1}{\sqrt{1+\alpha^{2}}} \cos \left(2\left(\theta-\theta_{e}\right)\right)\right)
$$

where $\theta_{e}$ is expressed explicitly versus $\alpha$ by (9).

\section{Appendix B: System of ODE for imposed stress}

Assuming a strictly positive apparent viscosity, we have $\operatorname{sgn}(\dot{\gamma}(t))=\operatorname{sgn}\left(\sigma_{x y}(t)\right)$ for all time $t \geqslant 0$ and relation (7e) leads to the following explicit expression of the shear rate $\dot{\gamma}$ versus the given shear stress $\sigma_{x y}$ and the unknown $\gamma_{e, x y}$ :

$$
\dot{\gamma}(t)= \begin{cases}\frac{\sigma_{x y}(t)}{\eta+\eta_{e}\left(\alpha \operatorname{sgn}\left(\sigma_{x y}(t)\right) \gamma_{e, x y}(t)+\beta \gamma_{e, x y}^{2}(t)\right)} & \text { when } \sigma_{x y}(t) \neq 0 \\ 0 & \text { otherwise }\end{cases}
$$

This expression of the shear rate $\dot{\gamma}$ is replaced in (7a)-(7b) and we then obtain a nonlinear ordinary differential equations (ODE) in terms of the two unknowns $\gamma_{e, x y}$ and $\gamma_{e, x x}$. These 
EDO are closed by two initial conditions $\gamma_{e, x y}(0)=\operatorname{sgn}\left(\sigma_{x y}(0)\right) \alpha^{-1}$ and $\gamma_{e, x x}(0)=2 \alpha^{-2}$. For the shear reversal, $\sigma_{x y}(0)$ is chosen and $\sigma_{x y}(t)=-\sigma_{x y}(0)$ for all $t>0$. For the shear reversal experiments of Blanc [39] with an imposed torque $T_{m}, \sigma_{x y}(0)$ is given in table I. After computation of $\gamma_{e, x y}$ and $\gamma_{e, x x}$, the rate of deformation $\dot{\gamma}(s)$ is computed from (B1) and finally, the deformation $\gamma(t)$ is obtained by a numerical integration as $\int_{0}^{t}|\dot{\gamma}(s)| \mathrm{d} s$. 